\title{
Research on the onboard auto-test system for track circuit compensating capacitors
}

\author{
H. Zhao, Y. Liu \& B. Liu \\ School of Electronics and Information Engineering, \\ Beijing Jiaotong University, China
}

\begin{abstract}
A kind of onboard auto-test system was proposed so as to detect the track circuit compensating capacitors in real time. The system model and design were based on the resonance current detection method. The data acquisition card generated the actuating sine signal first, which was amplified and coupled onto the rail. Two wheels of the test vehicle, rail lines between and the capacitor connected between the rails formed a closed LC loop. If the actuating signal frequency coincides with the inherent resonance frequency of the loop, a reactive current will be produced within the loop. After the current is sampled and further processed, the capacitor value can be estimated. The system principle and architecture are described in detail. Experiments show the invalid capacitors can be efficiently detected and located in real time.
\end{abstract}

Keywords: resonance current detection method, compensating capacitor, onboard auto-test system, track circuit, data acquisition.

\section{Introduction}

Audio frequency jointless track circuits, i.e. UM71 and ZPW-2000A, are now widely used in the railway autoblock systems in China. As for ZPW-2000A, rail lines act as the transmission media for dedicated FSK signal which carried the vital autoblock information used for train control. Modeled as a kind of distributed transmission line with inductive characteristic impedance, rail lines are not a good transmission media for audio frequency, unfortunately. In order to weaken the negative effect of the inductive rail and prolong the transmission length of track circuit, compensating capacitors are evenly spaced and installed between the rail lines, as shown in Fig. 1. 


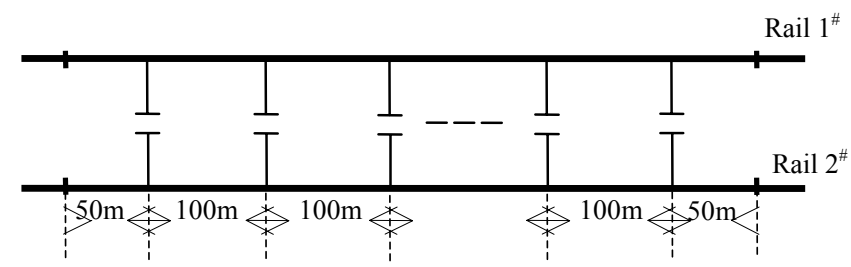

Figure 1: Compensating capacitor installation between rail lines.

\begin{tabular}{|c|c|c|c|c|}
\hline$\frac{1.7 \mathrm{KHz}}{55 \mu \mathrm{F}}$ & $\frac{2.3 \mathrm{KHz}}{46 \mu \mathrm{F}}$ & $\frac{1.7 \mathrm{KHz}}{55 \mu \mathrm{F}}$ & $\frac{2.3 \mathrm{KHz}}{46 \mu \mathrm{F}}$ & \\
\hline & & & & Line $1^{\#}$ \\
\hline $2.0 \mathrm{KHz}$ & $\underline{2.6 K H}$ & $2.0 \mathrm{KHz}$ & $2.6 \mathrm{KH}:$ & \\
\hline $50 \mu F$ & $40 \mu F$ & $50 \mu F$ & $\overline{40 \mu F}$ & \\
\hline
\end{tabular}

Figure 2: Compensating capacitor and carrier frequency configuration for Line $1^{\#}$ and Line $2^{\#}$ in Chinese railway.

For double lines, Line $1^{\#}$ and Line $2^{\#}$ use FSK modulated signal with different pair of carrier frequency. Besides that, the frequency is configured alternatively, as shown in Fig. 2. Hence, different compensating capacitor values should be used and configured correspondingly to match the different carrier frequency [1].

However, the evenly spaced compensating capacitors bring about another boring problem, i.e. the normal detection and maintenance of these capacitors is rather costly. The need for efficient onboard auto-test system is urgent.

Ref. [2] proposed a kind of compensation capacitor detection method based on wavelet analysis method by making use the inducting voltage curve received by cab signal receiving coils. But, this method can only judge whether the capacitor is fully invalid or not. Furthermore, it is only effective when the track circuit ballast impedance is approximately $1 \sim 3$ ohm.km. As the ballast impedance is often influenced by weather, geography conditions and other environmental factors and varies from $1 \sim 300 \mathrm{ohm} . \mathrm{km}$, the method is hardly to be used to guide maintenance.

At present, test vehicle has been the important measure for the online detection and maintenance of railway autoblock equipment. In this paper, a new kind of compensating capacitor auto-test system equipped on the test vehicle is put forward based on the resonance current detection method. 


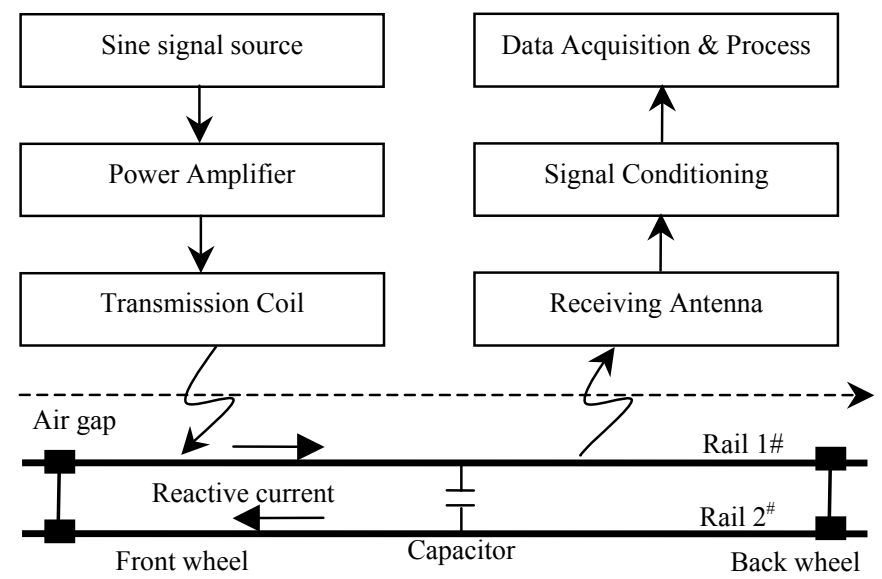

Figure 3: Principle of the resonance current detection method.

\section{Principle and system architecture}

\subsection{Resonance current detection method}

As shown in Fig. 3, a sine signal generator and a power amplifier are fixed on the test vehicle. Actuating sine signal is generated by the signal generator, amplified by the power amplifier, and then transmitted to the transmission coil installed under the vehicle. As the vehicle wheels and the rail lines between formed a closed loop, the magnetic field caused by the actuating signal will surely produce reactive current within the rail lines even though the current magnitude is relatively small. While the test vehicle passes the rail lines equipped with compensating capacitor connected in between, the above closed loop changes to an equivalent LC loop. Supposed that the actuating signal frequency is close to or equal to the resonance frequency of the LC loop, the reactive current will be enlarged to great extent.

The receiving antenna installed above the rail sense the current, transform it to proportional reactive voltage, and then transmit it to the signal conditioning unit. The data acquisition and process unit located backward analyzes the magnitude alteration of the reactive voltage and makes judgment whether the vehicle has passed a compensating capacitor. Capacitor value can be further calculated as the whole system is carefully scaled.

\subsection{System architecture}

Fig. 4 shows the installation position of dedicated test equipment under the test vehicle. A pair of transmission coil (TC1 and TC2) is fixed behind the front wheels, whereas another pair of receiving antenna (RA1 and RA2) is fixed before the back wheels. Speed sensor installed on one front wheel is used for train positioning. 


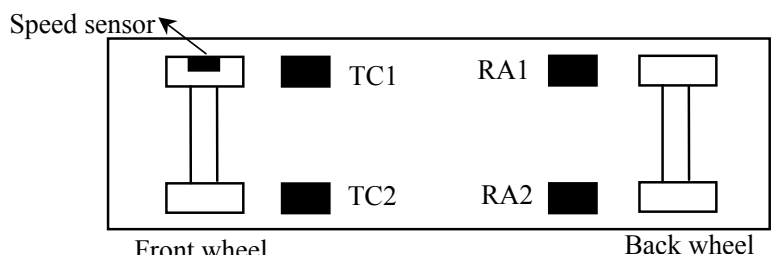

Figure 4: Installation position of dedicated test equipment under the test vehicle.

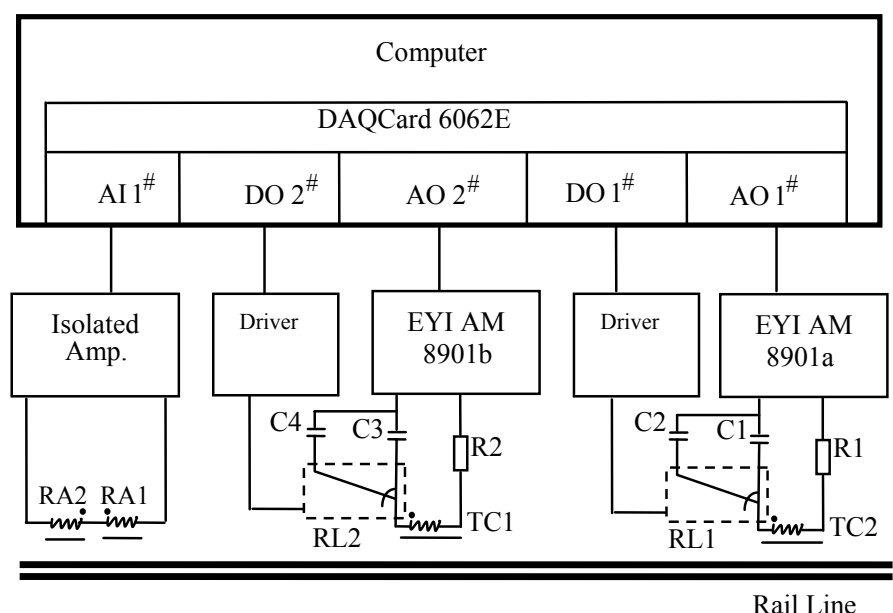

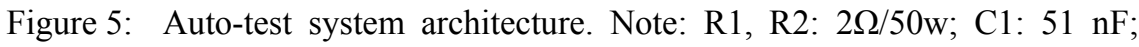
C2: $12 \mathrm{nF}$; C3: $72 \mathrm{nF}$; C4: nF; TC1, TC2: $10 \mathrm{mH}, \mathrm{Q}=21$; RA1, RA2: $63 \mathrm{mH}, \mathrm{Q}=5$.

Fig. 5 shows the architecture of the auto-test system. Computer and the data acquisition card of NI DAQCard 6062E are the key components of the system. The analog output channels $\mathrm{AO}^{\#}$ and $\mathrm{AO}^{\#}$ generate actuating sine signal, which is amplified by power amplifier EYI Am8901 and transmitted to the transmission coil TC1 and TC2 respectively. Within each AO channel, resistors $\mathrm{R} 1$ and R2 are used to limit the loop current; whereas capacitors $\mathrm{C} 1 \sim \mathrm{C} 4$ are used to counteract the inductive effect of transmission coil TC1 and TC2, improving the output current.

The signal generated by $\mathrm{AO}^{\#}$ is used to actuate the equivalent LC loop with compensating capacitor value of $40 \mathrm{e}-6 \mathrm{~F}$ or $46 \mathrm{e}-6 \mathrm{~F}$; while the signal generated by $\mathrm{AO}_{2}{ }^{\#}$ is used to actuate the LC loop with capacitor value of $50 \mathrm{e}-6 \mathrm{~F}$ or $55 \mathrm{e}-6$ $\mathrm{F}$. The two AO channels work at the same time, facilitating the simultaneous capacitor detection of 40e-6 F and 50e-6 F for Line $1^{\#}$ and $46 \mathrm{e}-6 \mathrm{~F}$ and $55 \mathrm{e}-6 \mathrm{~F}$ for Line $2^{\#}$.

The digital output channels $\mathrm{DO}^{\#}$ and $\mathrm{DO} 2^{\#}$ operate the power relays RL1 and RL2, making $\mathrm{C} 1$ and $\mathrm{C} 3$ connect with $\mathrm{C} 2$ and $\mathrm{C} 4$ wherever needed. 
The analog input channel $\mathrm{AI}^{\#}$ acquires the reactive voltage received by the receiving antenna RA1 and RA2 and isolated by ISO 124. RA1 and RA2 are connected each other in certain way so as to eliminate the interference caused by electrified traction current in electrified railway.

\subsection{Software implementation}

System software was developed under NI LabWindows /CVI, a kind of efficient C API for virtual instrumentation. System software functions are described as follows:

1) Generating actuating sine signal through AO channel, controlling the power relay through DO channel and selecting appropriate capacitor value $(\mathrm{C} 1 \sim \mathrm{C} 4)$

2) Acquiring the reactive voltage through AI channel, filtering the s Signal in frequency domain and calculating the rms value of the reactive voltage

3) Comparing the reactive voltage with the reference value, estimating the compensating capacitor value by polynomial curve fit method which is realize by the "PolyFit()" function in LabWindows/CVI.

4) Combining with the train positioning system, give the coordination of the capacitor detected.

\section{Experiment and data analysis}

A series of experiment are carried out while the auto-test system is setting up. The aim is to determine some obscure parameters and verify the validity of the resonance current detection method.

\subsection{Inherent resonance frequency determination experiment}

Although the compensating capacitor value and the rail impedance between the test vehicle wheels are predetermined, there still exist many obscure factors, including the wheel impedance, contact impedance between wheel and rail, the connecting impedance of capacitor with the rail, etc. Experiment should be done to determine the inherent resonance frequency of the equivalent LC loop. The normal static measurement method for system frequency response feature is used in the experiment [3]. The result is shown in Table 1.

The actuating signal frequency generated by AO channels of DAQCard 6062E can be fully determined according to Table 1 .

\subsection{Capacitor value sensitivity experiment}

Whether the reactive voltage is sensitive enough to the value change of the compensating capacitor within the equivalent LC loop is actually determined by the Q value of the LC loop itself. In the experiment, fixed rms value of actuating signal with specified frequency shown in Table 1 was firstly generated by Channel $\mathrm{AO}^{\#}$. The reactive voltage was then recorded as the capacitor value is changed. The result is shown in Table 2. 
Table 2 shows the reactive voltage is sensitive enough for the capacitor value measurement and the resonance current detection method is practical indeed.

\subsection{Capacitor position sensitivity experiment}

This experiment is used to check if the reactive voltage is sensitive enough to the position change of the compensating capacitor within the equivalent LC loop. The result is shown in Table 3.

Table 3 shows the largest reactive voltage appeared as the capacitor locates in center of the two wheel pairs. When the capacitor was moved outside the wheel pairs, the reactive voltage dropped rapidly. This shows the resonance current detection method is practical from another point of view.

Table 1: $\quad$ Result for inherent resonance frequency measurement.

\begin{tabular}{|c|c|c|c|c|}
\hline Compensating Capacitor (F) & $40 \mathrm{e}-6$ & $46 \mathrm{e}-6$ & $50 \mathrm{e}-6$ & $55 \mathrm{e}-6$ \\
\hline Inherent Resonance Freq. $(\mathrm{Hz})$ & 7050 & 6350 & 5950 & 5850 \\
\hline
\end{tabular}

Table 2: $\quad$ Result for capacitor value sensitivity experiment.

\begin{tabular}{|c|c|c|}
\hline $\begin{array}{l}\text { Actuating Signal } \\
\text { Frequency }(\mathrm{Hz})\end{array}$ & Compensating capacitorF) & Reactive Voltage $(\mathrm{mV})$ \\
\hline \multirow{6}{*}{7050} & None & 65.3 \\
\hline & 21.4 e- 6 & 98.7 \\
\hline & 26.2 e- 6 & 113.4 \\
\hline & 33 e- 6 & 133.8 \\
\hline & 40 e- 6 & 147.7 \\
\hline & 46 e- 6 & 90.0 \\
\hline \multirow{6}{*}{6350} & None & 57.4 \\
\hline & $21.4 \mathrm{e}-6$ & 77.9 \\
\hline & 26.2 e- 6 & 86.9 \\
\hline & 33 e- 6 & 103.0 \\
\hline & 40 e- 6 & 118.2 \\
\hline & 46 e- 6 & 135.3 \\
\hline \multirow{6}{*}{5950} & None & 53.8 \\
\hline & 26.2 e- 6 & 74.7 \\
\hline & 33 e-6 & 85.2 \\
\hline & 40 e- 6 & 96.1 \\
\hline & $46 \mathrm{e}-6$ & 107.5 \\
\hline & 50 e- 6 & 117.8 \\
\hline \multirow{6}{*}{5850} & None & 52.9 \\
\hline & 33 e- 6 & 80.2 \\
\hline & 40 e- 6 & 89.7 \\
\hline & $46 \mathrm{e}-6$ & 102.6 \\
\hline & 50 e- 6 & 112.3 \\
\hline & 55 e- 6 & 112.6 \\
\hline
\end{tabular}


Table 3: $\quad$ Result for capacitor position sensitivity experiment.

\begin{tabular}{|c|l|l|l|l|l|l|}
\hline Position* & 1 & 2 & 3 & 4 & 5 & 6 \\
\hline Reactive voltage $(\mathrm{mV})$ & 67.5 & 107.2 & 122.3 & 143.1 & 120 & 101.3 \\
\hline
\end{tabular}

* The position is shown as in the following figure.

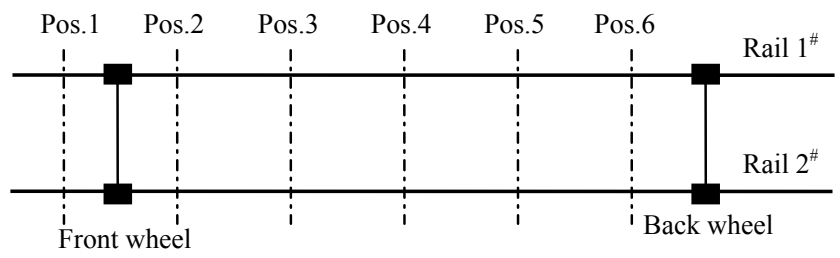

\section{Conclusion}

The onboard auto-test system of track circuit compensation capacitor is described in the paper. Conclusions are shown as follows:

1) Experiments show the resonance current detection method proposed in the paper is valid and efficient.

2) The compensating capacitor value can be estimated in real time, regardless the ballast impedance and other environmental factors.

3) Combining with the train positioning system, the coordination of the compensating capacitors under test can be provided.

4) Commercial data acquisition and virtual instrumentation techniques are applied in the auto-test system, ensuring the development efficiency and test accuracy.

\section{References}

[1] H. Zhao, Y. Zhang, and X. Wang, "Study on Several Problems of Track Circuit with Compensating Capacitors," Journal of Railways, China, vol. 20, no. 4, pp. 77-81, August 1998.

[2] L. Zhao, B. Liu, and X. Xu, "Using the Wavelet Analysis based Noise Canceling Technique to Detect The Integrity of The Compensating Capacitors of UM71 Track Circuit," IEEE 2005 International Symposium on Microwave, Antenna, Propagation and EMC Technologies for Wireless Communications Proceedings, $3^{\text {rd }}$ ed., China, vol. 2, pp.1263-1269, August 2005.

[3] H. Jiang, X. Sun, Electronic Measurement, Beijing: China Metrology Publishing House, 1988. 